\title{
Servidores de preprints: um caminho para a ciência aberta
}

\author{
PRÍNCIPE, Eloísa ${ }^{1}$ \\ ${ }^{1}$ Instituto Brasileiro de Informação em Ciência e Tecnologia (Ibict) - Brasil \\ ORCID ID: https://orcid.org/0000-0002-1540-0854
}

\begin{abstract}
Resumo
Descreve a evolução dos repositórios de preprints e suas principais características, destacando os seus pontos fortes e críticos. Apresenta exemplos de servidores de preprints no exterior e no Brasil. Conclui indicando a necessidade de ações fundamentadas e norteadoras tanto em relação aos autores e às revistas científicas quanto às agências de fomento e pesquisa, para a sua ampla adoção, considerando as particularidades e especificidades de cada área do conhecimento. $\mathrm{O}$ uso e ampla divulgação do preprint como recurso para acelerar a disseminação do conhecimento podem ser benéficos para a carreira acadêmica do pesquisador e para a sociedade em geral, ampliando e consolidando os caminhos da Ciência Aberta.
\end{abstract}

Palavras-chave: Ciência Aberta. Comunicação Científica. Preprint.

\begin{abstract}
Preprints servers: a path to open science

Describes the evolution of the preprints repositories and their main characteristics, highlighting their strong and critical points. Presents examples of preprint servers abroad and in Brazil. It concludes by indicating the need for reasoned and guiding actions related to its wide adoption, by authors and scientific journals, as well as to research funding agencies, considering the particularities and specificities of each area of knowledge. The use and wide disclosure of the preprint as a resource to accelerate the dissemination of knowledge can be beneficial for the researcher academic career and for society in general, expanding and consolidating the paths of Open Science.
\end{abstract}

Keywords: Open Science. Scholarly Communication. Preprint.

\section{Resumen}

Servidores de preprints: un camino para la ciencia abierta

Describe la evolución de los repositorios de preprints y sus principales características, destacando sus puntos fuertes y críticos. Presenta ejemplos de servidores de preprints en el exterior y en Brasil. Se concluye indicando la necesidad de acciones firmes y orientadoras tanto en relación con los autores y revistas científicas como con las agencias de desarrollo e investigación, para su amplia adopción, considerando las particularidades y especificidades de cada área de conocimiento. El uso y la amplia difusión del preprint como medio de acelerar la divulgación del conocimiento puede ser beneficios para la carrera académica del investigador y para la propia sociedad en general, ampliando y consolidando los caminos de la Ciencia Abierta.

Palabras clave: Ciencia Abierta. Comunicación Científica. Preprint. 


\section{Compreendendo o cenário}

Uma das práticas da ciência que vem tomando cada vez mais fôlego na esfera do movimento da ciência aberta, refere-se à publicação de preprints. De acordo com o Committee of Publication Ethics (COPE), um preprint “[...] é um manuscrito científico depositado pelo(s) autor(es) numa plataforma abertamente acessível, geralmente antes ou em paralelo com o processo de revisão pelos pares.” (COPE, 2018, em linha). O seu principal objetivo é agilizar o processo da comunicação científica, de maneira aberta, transparente, imediata e ampla, contrapondo-se ao sistema tradicional que se apresenta fechado, moroso e restrito na avaliação dos artigos pelos pares. Esses papers são depositados em servidores ou repositórios de preprints. A principal razão para a adoção desta modalidade de publicação "[...] é o estabelecimento da precedência de descobertas, a diminuição do tempo para comunicar os resultados de pesquisa e a oportunidade de receber comentários e feedback sobre o trabalho depositado no servidor." (SPINAK, 2019).

No ambiente eletrônico ${ }^{1}$, em 1991, foi lançada a primeira plataforma de preprint - o arXiv, por Paul Ginsparg, no Laboratório Nacional de Los Alamos ${ }^{2}$, Estados Unidos, para a área de física e, posteriormente, estendida a campos relacionados, como matemática, astronomia, ciência da computação, ciência não-linear, biologia quantitativa, estatística, finanças quantitativas, economia e engenharia elétrica e de sistemas. Ao longo dessas três décadas, várias plataformas de preprints foram criadas: gerais (Preprints.org, SSRN, Research Square) ou temáticas (ChemRxiv, medRxiv, LawArXiv, psyArXiv), mas também nacionais (FrenXiv, AfricArxiv, ChinaXiv), indicando uma aceitação dessa modalidade de publicação por parte dos autores, considerando o crescimento substancial no número de preprints depositados nas diferentes plataformas ${ }^{3}$, de financiadores ${ }^{4}$ e de editoras de revistas científicas ${ }^{5}$, que utilizam também essas plataformas para identificar textos relevantes que, potencialmente, são adequados para publicação em suas revistas. Os autores são convidados a submeter seus manuscritos (preprints) à publicação ${ }^{6}$. Atualmente, a possibilidade e o

\footnotetext{
${ }^{1}$ Antes, nas décadas de 1950 e 1960, o Centro Brasileiro de Pesquisas Físicas (CBPF) e os National Institutes of Health/The Information Exchange Groups Experiment (1961-1966) lançaram iniciativas de preprints impressos. O do Brasil continua até hoje em formato eleltrônio e conta com a participação de pesquisadores externos ao CBPF.

${ }^{2}$ Hoje armazenado e operado pela Cornell University.

${ }^{3} \mathrm{O}$ crescimento mensal de depósitos de preprints em diferentes plataformas pode ser visualizado no PrePubMed. Disponível em: http://www.prepubmed.org/monthly_stats/. Acesso em: 30 ago. 2020.

${ }^{4}$ Em 2017, a Wellcome Trust anunciou que passou a aceitar preprints nas referências bibliográficas dos projetos por ela apoiados e os National Institutes of Health (NIH) anunciaram que as propostas de financiamento e relatórios de auxílios concedidos dirigidos à instituição estavam autorizadas - e encorajadas - a citar preprints. (NASSI-CALÒ, 2017).

${ }^{5}$ The Journal of Clinical Investigation, Physical Review, Physics Today, Science, Memórias do Instituto Oswaldo Cruz, Revista da Sociedade Brasileira de Medicina Tropical.

${ }^{6}$ Algumas revistas já criaram a função de "editor de preprint" para realizar essa "busca" por manuscritos de qualidade nos servidores de preprint. (BARSH et al. 2016). Exemplos: Proceedings of the Royal Society B, PLOS Genetics, Open Biology, Genome Biology.
} 
interesse em publicar no formato de preprint aumenta a cada dia, com maior ou menor incidência em praticamente todas as áreas científicas. (FERREIRA; SERPA, 2018) ${ }^{7}$. O número de preprints depositados no arXiv, de setembro de 1991 (58), data de seu lançamento, a agosto de $2020(11.715)^{8}$, alcança 1.757.455 preprints $^{9}$. Em geral, os repositórios destacam, nas páginas dos preprints, que esses documentos são "relatórios preliminares que não foram revisados por pares", alertando para o seu uso, pois não devem ser considerados como conclusivos (KIRKHAM et al., 2020). Em outros casos, é inserida uma marca d'água nas páginas, informando que o documento não é revisado. Antes de disponibilizar o preprint, os servidores fazem uma triagem, visando identificar, principalmente, problemas éticos:

Para ser publicado nos servidores de preprint medRxiv e bioRxiv, um artigo deve passar por uma avaliação básica que busca identificar plágio, conteúdo ofensivo, conteúdo não científico e material que possa representar risco à saúde. Os examinadores não avaliam os métodos, conclusões ou a qualidade de um artigo. (ORDWAY, 2020, em linha).

O preprint recebe um número DOI - Digital Object Identifier e os servidores permitem o seu versionamento, mas os autores não podem retirar os documentos já depositados. O preprint fica disponível para acesso por qualquer pessoa e os leitores podem apresentar comentários, que são inseridos na plataforma junto ao documento, por outro lado, os autores podem aproveitar esses comentários e atualizar o preprint. O resultado da pesquisa de Rubén Alvarez e Caregnato (2017, em linha) demonstrou a aceitabilidade dos preprints pelos editores das revistas científicas da área de Física de Altas Energias (FAE), concluindo sobre "[...] a potencialidade dos preprints como canal precursor de difusão de resultados científicos visto que $70 \%$ das submissões [preprints] foram em seguida absorvidas pelas principais revistas da FAE.” Embora o número de preprints venha aumentando progressivamente, o modelo enfrenta resistências que podem ser exemplificadas, segundo Souza (2020, em linha), pelas seguintes preocupações:

[...] qualidade dos documentos submetidos aos repositórios; a existência de uma avaliação prévia; a total responsabilidade do autor sobre o que é publicado; a publicação de erros metodológicos e estatísticos; a pouca interatividade nos repositórios que possibilitam comentários; a legitimidade dos documentos para recuperação em revisões e estudos documentais/bibliométricos; a competitividade que poderia ser fomentada; o novo papel para os periódicos diante da mudança do sistema duplo-cego e o risco de roubo de ideias.

\footnotetext{
${ }^{7}$ Não se pode deixar de mencionar que a pandemia da covid-19, em 2020, iniciada no final de 2019 na China, impulsionou o número de depósitos e de dowloadings desses textos em diferentes servidores de preprint. O bioRxiv (Ciências Biológicas) e o medRxiv (Ciências da Vida), servidores mais utilizados na pandemia, registraram juntos, 8433 artigos $^{7}$, sendo 1785 depositados no bioRxiv e 6648 no medRxiv. Dados de 5 de setembro de 2020. Disponível em: https://connect.biorxiv.org/relate/content/181. Acesso em: 5 set. 2020.

${ }^{8}$ Dados de 6 de setembro de 2020.

${ }^{9}$ Dados de 6 de setembro de 2020.
} 


\section{Cenário brasileiro}

No Brasil, merecem destaque duas iniciativas pioneiras e recentes: o SciELO Preprint ${ }^{10}$ e o EmeRI - Emerging Researcher Information ${ }^{11}$.

Como parte do alinhamento do Programa SciELO com a Ciência Aberta, foi lançado, em 7 de abril deste ano, o servidor SciELO Preprint, operado pelo Open Preprint Systems (OPS), software de código aberto, desenvolvido pelo Public Knowledge Project (PKP) (SCIELO, 2020). A plataforma, implementada em três idiomas, está organizada nas seguintes áreas temáticas: Ciências Agrárias, Ciências Sociais Aplicadas, Ciências Biológicas, Engenharia, Ciências Exatas e da Terra, Ciências da Saúde, Ciências Humanas, Linguística, Literatura e Artes e Interdisciplinar. Após passar por uma triagem básica, realizada pelos editores de área do manuscrito, é atribuído um DOI ao preprint e, então, é disponibilizado na ordem em que foi aprovado para publicação, sob a licença "CC BY" e sujeito à comentários. Depois de publicados, os preprints não podem ser removidos pelos autores. O servidor SciELO Preprints removerá os preprints sempre que for detectada uma violação da ética (SCIELO PREPRINT, 2020). A busca na plataforma pode ser feita por termos livres, e os filtros avançados permitem a delimitação temporal e por autor. Hoje, o número de papers depositados chega a $504^{12}$. Todas as áreas estão cobertas, sendo a área das Ciências da Saúde a que mais se destaca, com71\%. As Ciências Humanas e Sociais Aplicadas apresentam 10\% cada uma. As Engenharias apresentam apenas um preprint depositado.

O EmeRI foi lançado oficialmente no dia 20 de maio. Seu desenvolvimento foi fruto da parceria entre a Associação Brasileira de Editores Científicos (Abec Brasil) e o Instituto Brasileiro de Informação em Ciência e Tecnologia (Ibict). Foi desenvolvido na plataforma DSpace ${ }^{13}$ e os documentos são organizados em comunidades, coleções e itens: revistas científicas é a comunidade principal e cada revista é uma coleção, que abriga, como itens, os preprints depositados ${ }^{14}$. O EmeRI “[...] é um indexador de revistas acadêmicas/científicas, que oferece um serviço para publicação das submissões por elas recebidas, na forma de preprints, imediatamente após sua aprovação na revisão de conformidade (desk review)" ${ }^{\prime 15}$. Diferentemente da maioria dos servidores de preprint, no EmeRI quem deposita o paper não é o autor, mas sim o editor da revista à qual foi submetido o artigo. Assim, apenas as revistas cadastradas nessa plataforma é que podem depositar preprints. A busca facetada no servidor pode ser feita pelo idioma do preprint, país de publicação da revista, área de

\footnotetext{
${ }^{10}$ Disponível em: https://preprints.scielo.org/index.php/scielo. Acesso em: 30 ago. 2020.

${ }^{11}$ Disponível em: https://preprints.ibict.br/sobrePreprint.jsp. Acesso em: 30 ago. 2020.

${ }^{12}$ Dados de 6 de setembro de 2020.

${ }^{13}$ Software de código-fonte aberto.

${ }^{14}$ Disponível em: https://preprints.ibict.br/perguntasFrequentes.jsp. Acesso em: 30 ago.2020.

${ }^{15}$ Disponível em: https://preprints.ibict.br/perguntasFrequentes.jsp\#TD20. Acesso em: 30 ago. 2020.
} 
conhecimento da revista e pelos serviços de informação universais. Atualmente, oito revistas estão indexadas no EmeRI.

\section{Considerações finais}

Os preprints vêm demarcando uma nova forma de comunicar e compartilhar a ciência. Sua aceitação pode ser atestada pelo número de servidores criados em áreas específicas e gerais e pelo número cada vez maior de depósitos realizados nessas plataformas. Entretanto, sua efetiva adoção depende de ações fundamentadas e norteadoras tanto em relação aos autores e às revistas científicas quanto às agências de fomento e pesquisa, considerando as particularidades e especificidades de cada área do conhecimento. O uso e a ampla disseminação do preprint como recurso para acelerar a disseminação de conhecimentos podem ser benéficos para a carreira acadêmica do pesquisador e para a própria sociedade em geral, ampliando e consolidando os caminhos da Ciência Aberta.

\section{Referências}

BARSH, Gregory S., et al. Bringing PLOS Genetics editors to preprint servers. PLoS Genetics, v. 12, n. 12, Dec. 2016. Disponível em: https://journals.plos.org/plosgenetics/article?id=10.1371/journal.pgen.1006448. Acesso em: 27 ago. 2020.

COPE. COPE Discussion document: Preprints. Mar. 2018. Disponível em: https://publicationethics.org/files/u7140/COPE_Preprints_Mar18.pdf. Acesso em: 1 set. 2020.

FERREIRA, Carlos Miguel; SERPA, Sandro. The importance of preprint in scientific publication. The Journal of Social Sciences Research, v. 4, n. 12, p. 642-647, 2018. Disponível em: https://run.unl.pt/bitstream/10362/99544/1/The_Importance_of_Preprint_in_Scientific_Publication .pdf. Acesso em: 22 ago. 2020.

KIRKHAM, Jamie J., et al. A systematic examination of preprint platforms for use in the medical and biomedical sciences setting. BioRxiv: The Preprint Server for Biology, 2020. Disponível em: https://www.biorxiv.org/content/10.1101/2020.04.27.063578v1. Acesso em: 5 set. 2020.

NASSI-CALÒ, Lilian. Propostas de financiamento a pesquisa enviadas ao NIH podem citar preprints. SciELO em Perspectiva, 2017. Disponível em:

https://blog.scielo.org/blog/2017/05/17/propostas-de-financiamento-a-pesquisa-enviadas-ao-nihpodem-citar-preprints/\#.X1p24X1KhPY. Acesso em: 27 ago. 2020.

ORDWAY, Denise-Marie. Cobertura de preprints de pesquisa biomédica em meio ao coronavírus: 6 coisas a saber. [em linha]. SciELO em Perspectiva, 2020. Disponível em: scielo.org/blog/2020/04/15/cobertura-de-preprints-de-pesquisa-biomedica-em-meio-aocoronavirus-6-coisas-a-saber-originalmente-publicado-no-journalists-resource-em-abril-2020. Acesso em: 30 ago. 2020. 
RUBÉN ALVAREZ, Gonzalo; CAREGNATO, Sônia Elisa. Preprints na comunicação científica da Física de Altas Energias: análise das submissões no repositório arXiv (2010-2015).

Perspectivas em Ciência da Informação, Belo Horizonte, v. 22, n. 2, p. 104-117, jun. 2017.

Disponível em: https://www.scielo.br/scielo.php?script=sci_arttext\&pid=S1413-

99362017000200104\&lng=pt\&nrm=iso. Acesso em: 27 ago. 2020.

SCIELO. SciELO Preprints em operação. SciELO em Perspectiva, 2020. Disponível em: https://blog.scielo.org/blog/2020/04/07/scielo-preprints-em-operacao/\#.X1p3p31KhPY. Acesso em: 1 set. 2020.

SCIELO PREPRINT. About the Server. 2020. Disponível em: https://preprints.scielo.org/index.php/scielo/about. Acesso em: 1 set. 2020.

SOUZA, Jonathan Renan da Silva. Preprints na ciência brasileira: considerações sob a ótica da Enfermagem. BlogRev@Enf, 2020. Disponível em: https://blog.revenf.org/2020/05/15/preprintsna-ciencia-brasileira-consideracoes-sob-a-otica-da-enfermagem/. Acesso em: 1 set. 2020.

SPINAK, Ernesto. Acelerando a comunicação científica via preprints. SciELO em Perspectiva, 2019. Disponível em: https://blog.scielo.org/blog/2019/10/04/acelerando-a-comunicacaocientifica-via-preprints/. Acesso em: 29 ago. de 2020.

SPINAK, Ernesto. O que é este tema dos preprints? SciELO em Perspectiva, 2016. Disponível em: https://blog.scielo.org/blog/2016/11/22/o-que-e-este-tema-dos-preprints/. Acesso em: 1 set. 2020.

\section{${ }^{1}$ Eloísa Príncipe (Rio de Janeiro, RJ, Brasil)}

Doutora e Mestre em Ciência da Informação pela Universidade Federal do Rio de Janeiro (UFRJ). Bacharel em Biblioteconomia e Documentação pela Universidade Federal Fluminense (UFF). Professora do Programa de PósGraduação em Ciência da Informação (convênio UFRJ-IBICT). Tecnologista do Instituto Brasileiro de Informação em Ciência e Tecnologia (IBICT). Sócia e membro do Conselho Deliberativo da Associação Brasileira de Editores Científicos (ABEC Brasil) da qual já foi membro da Diretoria, por dois mandatos, além de participar de seu Conselho Deliberativo. Ex-Editora Adjunta da revista Tendências da Pesquisa Brasileira em Ciência da Informação, publicada pela Associação Nacional de Pesquisa e Pós-Graduação em Ciência da Informação (ANCIB).

príncipe@ibict.br

Avaliador(a) responsável: Eli Lopes da Silva

\section{Como citar este artigo:}

PRÍNCIPE, Eloísa. Servidores de preprints: um caminho para a ciência aberta. In: ABEC Meeting Live, 2020. Anais... São Paulo: Associação Brasileira de Editores Científicos, 2020. DOI: http://dx.doi.org/10.21452/abecmeeting2020.07 